\title{
Surgical pitfalls of minimally invasive direct coronary artery bypass procedure from the viewpoint of a surgeon in the learning curve
}

\author{
Bilgin Emrecan'1, Ahmet Coșkun Özdemir² \\ 1Department of Cardiovascular Surgery, Pamukkale University, Denizli, Turkey \\ 2Department of Cardiovascular Surgery, Trakya University, Edirne, Turkey
}

\begin{abstract}
Introduction: Minimally invasive direct coronary artery bypass grafting (MIDCAB) offers arterial revascularization of the left anterior descending (LAD) coronary artery especially in lesions unsuitable for percutaneous coronary interventions. By avoidance of sternotomy and cardiopulmonary bypass its invasiveness is less than that of conventional bypass surgery.

Aim: We in this study discuss our surgical experience in the MIDCAB procedure.

Material and methods: Thirteen patients were operated on with the MIDCAB procedure. The inclusion criteria for $M I D C A B$ were pure $L A D$ disease totally occluded or severely stenotic. Patient demographics and preoperative and postoperative data were analyzed.

Results: Mean age of the patients was $60.0 \pm 8.6$ years. Patients' preoperative and postoperative levels of cardiac $C K-M B$ (creatine kinase MB) were not significantly different $(p=0.993)$. However, cardiac troponin I $(p<0.001)$, hemoglobin $(p<0.001)$ and hematocrit $(p<0.001)$ were significantly different. No perioperative myocardial infarctions or cerebrovascular accidents were seen. The patients were discharged at a mean day of 4.77 with oral antiaggregant therapy. No mortality was seen in the study population.

Conclusions: Minimally invasive direct coronary artery bypass is associated with few perioperative complications. Minimally invasive direct coronary artery bypass in our experience is a very good option for single vessel LAD disease.
\end{abstract}

Key words: coronary artery disease, minimally invasive cardiac surgery.

\section{Introduction}

Coronary revascularization between the internal mammary artery and a coronary artery was described by Kolessov several decades ago [1]. Since the description of minimally invasive direct coronary artery bypass grafting (MIDCAB) by Calafiore et al. [2], surgical revascularization has been further developed. Many studies have proved several advantages of the technique besides its similar outcomes to the conventional coronary artery bypass grafting (CABG) or off-pump coronary artery bypass (OPCAB).

Minimally invasive direct coronary artery bypass can be regarded as an alternative to percutaneous coronary interventions $(\mathrm{PCl})$ in patients with proximal stenosis of the left anterior descending artery (LAD), especially patients for whom $\mathrm{PCl}$ is risky or impossible. Sometimes MIDCAB offers a good alternative especially for totally occluded LADs or in-stent stenotic LADs.

Bilgin Emrecan, Yunus Emre cad. No: 83/5, Kınıklı 20070, Denizli, Turkey, phone: +905054889916, e-mail: bilginemrecan@yahoo.com 


\section{Aim}

In the present study, we retrospectively studied the early postoperative and short-term results of the MIDCAB procedure. The surgical outcomes and surgical learning curve with its difficulties were discussed in the light of the literature.

\section{Material and methods}

Thirteen patients were operated on with the MIDCAB procedure from October 2010 to December 2011. The inclusion criteria for MIDCAB were pure LAD disease totally occluded or severely stenotic and patients for whom $\mathrm{PCl}$ is risky or impossible (Photo 1 ). The stenoses of the other vessels were not significant. In all patients LADs were antegradely or retrogradely filled by collateral circulation. All patients had stable or unstable angina pectoris unresponsive to medical treatment.

Patient demographics and preoperative and postoperative data were analyzed. Cardiac troponin I and creatine kinase $M B(C K-M B)$ were measured before surgery and $24 \mathrm{~h}$ after the surgery. Normal values of the cardiac markers were $0.00-0.4 \mathrm{mg} / \mathrm{dl}$ for cardiac troponin I and 0-24 U/I for CK-MB. Increase in CK-MB levels of more than $100 \mathrm{U} / \mathrm{l}$ was considered diagnostic of myocardial infarction.

\section{Surgical technique}

The patients were operated on under general anesthesia intubated with a double-lumen tube. Standard monitoring with 5-lead electrocardiogram (ECG), arterial blood pressure, central venous pressure, nasopharyngeal temperature and peripheral oxygen saturation were attained. A small 7-8 cm submammary left anterior thoracotomy from the fourth intercostal space was done (Photo 2). The pleural cavity was opened for harvesting of the arterial graft with the left lung deflated. The left internal mammary artery (LIMA) was harvested in a skeletonized fashion up to the level of the left subclavian vein. A thoracic wall tilting device (ThoraGate ${ }^{\mathrm{TM}}$ Rib Up Retractor for MIDCAB with flexible arm cardiac stabilizer, Geister, Germany) was used for LIMA harvesting. After completion of harvesting, the LIMA graft was rinsed with papaverine in order to prevent vasospasm.

After the pericardium had been opened starting from the antero-apical part of the phrenic nerve, $100 \mathrm{lU} / \mathrm{kg}$ of unfractionated heparin was administered.
After the LAD anastomosis site had been stabilized with the cardiac stabilizer, the artery was clamped with a bulldog clamp from proximal and distal sites of the arteriotomy. The arteriotomy field was kept free of blood by a humidified blower using fluid and carbon dioxide. If possible intracoronary shunt was applied and the bulldog clamps were taken. The anastomosis between LIMA and LAD was performed under direct vision with 7/0 polypropylene suture (Photo 3). Heparin was neutralized with protamine as the anastomosis was completed. A chest tube was placed in the left pleural cavity. The thoracotomy was closed after intercostal nerve blockage with bupivacaine. The double lumen intubation had been changed with a standard intubation tube

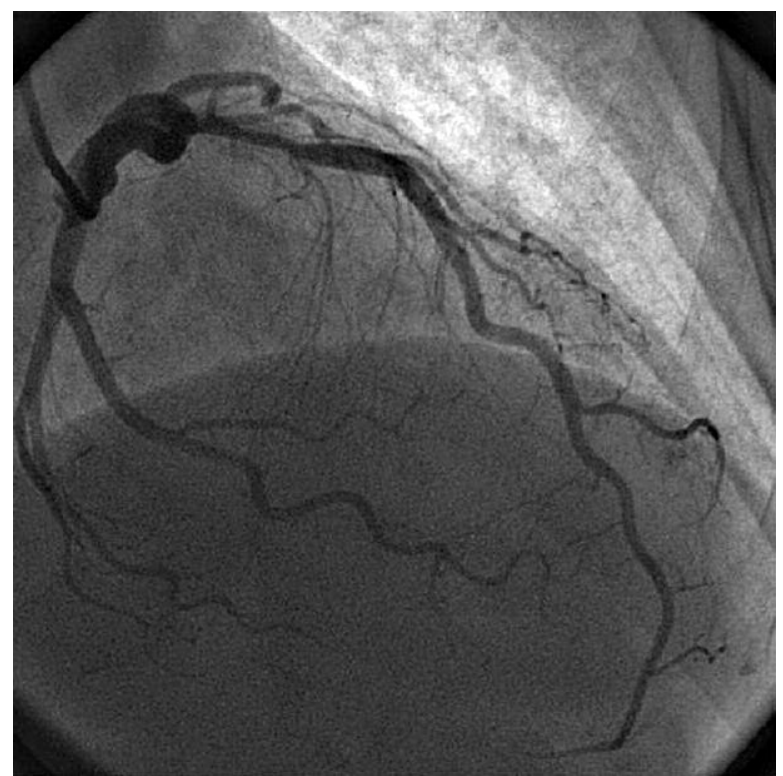

Photo 1. Preoperative coronary angiography showing proximal LAD lesion

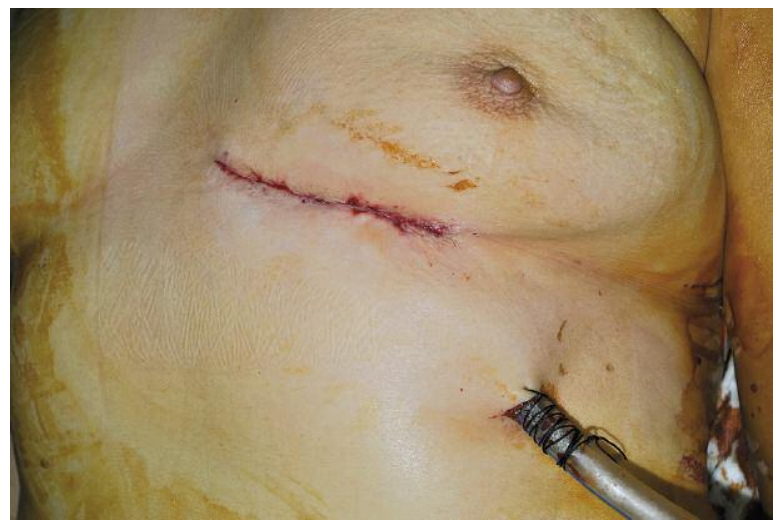

Photo 2. MIDCAB incision 


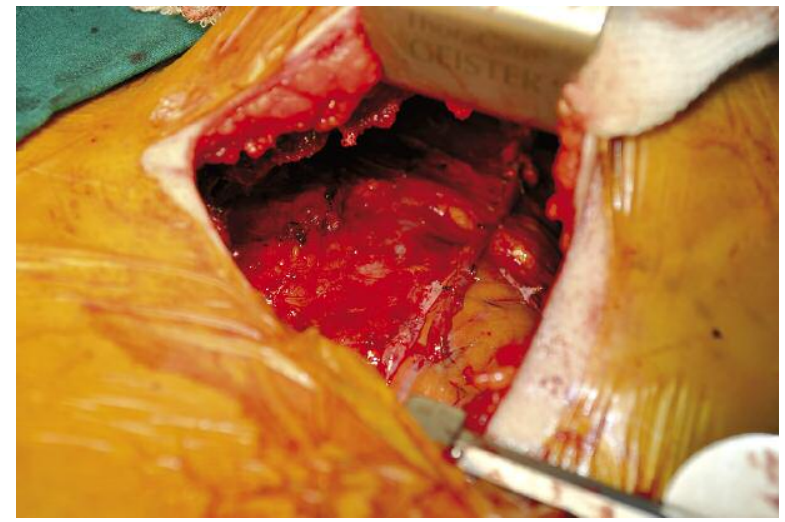

Photo 3. LIMA-LAD anastomosis completed

before the patient was taken to the intensive care unit.

\section{Statistical analysis}

The preoperative and postoperative data of the patients were analyzed. Statistical analysis was performed with SPSS statistical software version 17.0 (SPSS Inc.; Chicago, III). Continuous variables were expressed as mean \pm SD and were compared by means of the paired samples $t$ test. A value of $p$ less than 0.05 was considered to be statistically significant.

\section{Results}

We operated on 13 patients who had the inclusion criteria from October 2010 to December 2011. The male/female ratio was $8 / 5$. Mean age of the patients was $60.0 \pm 8.6$ years. The risk factors for coronary arterial disease were hypertension in 5 patients, smoking in 6 patients, hypercholesterolemia in $7 \mathrm{pa}$ tients, diabetes in 6 patients, peripheral arterial occlusive disease in 1 patient and family history of coronary artery disease in 2 patients. Four of the patients had chronic obstructive pulmonary disease. One patient had preoperative myocardial infarction 5 days before the surgery. Patients' data are presented in the Table I.

Patients' preoperative and postoperative levels of cardiac CK-MB were not significantly different $(p=0.993)$. However, the preoperative and postoperative levels of cardiac troponin I $(p<0.001)$, hemoglobin $(p<0.001)$ and hematocrit $(p<0.001)$ were significantly different. No perioperative myocardial infarctions or cerebrovascular accidents were seen. The increase in the troponin I levels were within the normal postoperative ranges.
One patient had prolonged tube thoracostomy due to air leakage from the pleural cavity which also prolonged the hospitalization to 8 days. One patient had local wound infection after discharge which was treated with antibiotics and local wound care. One patient had pleural effusion that needed pleural drainage with a needle in the late follow-up.

Patients had slightly low intubation $(4.38 \pm 1.19 \mathrm{~h})$ and intensive care unit follow-up (19.00 $\pm 4.18 \mathrm{~h}$ ) durations. The patients were discharged at a mean day of 4.77 with oral antiaggregant therapy. No mortality was seen in the study population. Patients were followed up at a mean of 9.84 months and they were free of angina in the postoperative period.

\section{Discussion}

The MIDCAB having been introduced constitutes an attractive surgical option for patients with one-vessel coronary artery disease of the LAD. It is also recommended for patients for a hybrid procedure, which involves revascularization of the $L A D$ and stenting of additional coronary arteries in patients with more advanced disease. Patients with three-vessel disease and concomitant malignancies, severely reduced lung function and overall reduced life expectancy may also be candidates for this procedure [3].

The MIDCAB is associated with significantly lower rates of repeat target vessel revascularizations [4-6]. These studies compared MIDCAB with bare metal stents. Thiele and co-workers published a randomized trial with a non-inferiority design which compared $P C I$ of the $L A D$ with drug-eluting stents and MIDCAB. The results were comparable concerning death and myocardial infarction but the rate of target vessel revascularization was still higher in the $\mathrm{PCl}$ group [7].

Although some apply ischemic preconditioning, we in our cases either used intracoronary shunts or just clamping the LAD in order to make a better visual field. Temporary occlusion anticipates the expectable events during anastomosis such as ST elevation, rhythm disturbances and hemodynamic compromise. These events are more likely in partially occluded target vessels and close to other coronary vessels. We did see ST elevations in some patients but these elevations did not cause enzymatically detected damage to the myocardium. In this context, why we did not use intracoronary shunt may be discussed. It is not a very usual visual field from a small 
Table I. Preoperative and postoperative data

\begin{tabular}{|c|c|c|c|c|c|}
\hline Variables & $N$ & Min. & Max. & Mean & Standard deviation \\
\hline EuroSCORE & 13 & 0.88 & 6.37 & 2.89 & 1.69 \\
\hline Left ventricular ejection fraction [\%] & 13 & 40 & 70 & 54.9 & 9.0 \\
\hline Age [years] & 13 & 44 & 71 & 60.0 & 8.6 \\
\hline Preoperative CK-MB [U/I] & 13 & 10 & 292 & 48.8 & 75.7 \\
\hline Postoperative 24th h CK-MB [U/l] & 13 & 28 & 120 & 48.6 & 27.8 \\
\hline Fasting blood glucose [mg/dl] & 13 & 89 & 406 & 149 & 86 \\
\hline Preoperative hematocrit [\%] & 13 & 35.4 & 43.6 & 39.4 & 2.6 \\
\hline Hematocrit at discharge [\%] & 13 & 27.6 & 37.4 & 32.0 & 3.0 \\
\hline Preoperative hemoglobin [g/dl] & 13 & 11.9 & 14.7 & 13.5 & 0.9 \\
\hline Hemoglobin at discharge $[\mathrm{g} / \mathrm{dl}]$ & 13 & 9.7 & 12.9 & 11.2 & 0.9 \\
\hline Preoperative troponin I [mg/dl] & 13 & 0.00 & 0.34 & 0.076 & 0.099 \\
\hline Postoperative $24^{\text {th }} \mathrm{h}$ troponin I [mg/dl] & 13 & 0.05 & 0.55 & 0.288 & 0.145 \\
\hline Transfusion (bag of blood) & 13 & 0 & 1 & 0.23 & 0.44 \\
\hline LAD diameter $[\mathrm{mm}]$ & 13 & 1.0 & 3.5 & 2.31 & 0.69 \\
\hline Extubation [h] & 13 & 3 & 6 & 4.38 & 1.19 \\
\hline Intensive care unit follow-up [h] & 13 & 14 & 28 & 19.00 & 4.18 \\
\hline Hospitalization [day] & 13 & 3 & 8 & 4.77 & 1.24 \\
\hline Follow-up [month] & 13 & 1 & 14 & 9.84 & 3.74 \\
\hline
\end{tabular}

$C K-M B$ - creatine kinase-MB, $L A D$ - left anterior descending artery

opening; thus to introduce a shunt is not that easy. Another aspect of the MIDCAB procedure is that every small surgical instrument like the shunts or bulldog clamps should be held with a suture material ligated to it because any material dropped in the surgical area may not be found and taken out through the small opening. At the very beginning we opened the thorax a little apart from the midline in order not to injure the LIMA but this incision made it difficult to reach the LIMA and of course the heart. So we also suggest a rear incision to the midline for better exposure.

A meta-analysis of 17 studies of MIDCAB showed early and late death rates as 1.3 and $3.2 \%$. At 6 month follow-up $3.6 \%$ of grafts were occluded and $7.2 \%$ had a significant stenosis, which resulted in $3.3 \%$ target vessel revascularization. The incidence of myocardial infarction was below $1 \%$. After a learning curve one could assume that the results of MIDCAB may get better [8]. The MIDCAB has long-term anastomotic patency rates comparable with those of open-chest LIMA-left-anterior descending artery (LAD) bypass [9]. Some authors stress that the quality of the MIDCAB procedure would remain high when performed in centers with a caseload adequate to allow surgeons to continuously sustain their skill level. In repeated in-stent stenosis, complete occlusion of the LAD, or lesions not suitable for stenting for anatomic reasons (complex type $\mathrm{C}$ stenosis or small vessels), the MIDCAB operation is offered as a good alternative [10].

Our experience showed that the duration of the operation has been shortened to approximately two thirds of the initial one. This is due to getting used to LIMA harvesting and due to learning how to use the equipment, which is slightly different than the conventional one. We also recommend skeletonizing the LIMA grafts in order to obtain longer 
grafts. For this purpose we recommend harvesting LIMA in a skeletonized fashion during the standard median sternotomy approaches to learn how to do it, unless the surgeon has harvested before. The LIMA flow may sometimes be low in case of a subclavian artery stenosis or occlusion. Routine examination of the blood pressure on both arms may to some extent rule out subclavian stenosis. A difference of blood pressure of more than $20 \mathrm{~mm} \mathrm{Hg}$ should be a clue for a cardiologist to supplement coronary angiography with aortic arch imaging. Should subclavian arterial stenosis be found, it has to be corrected before cardiac surgery [11].

The small number of cases is a real limitation in such studies. But surgeons like us in the learning curve may experience many difficulties in their initial cases. In 1 patient for instance we encountered an intramuscular course of LAD but this did not necessitate conversion to midline sternotomy. Excessive epicardial fat tissue may also make the target vessel difficult to find and the anastomosis difficult to do, although we did not meet such cases. Therefore for the learning curve patients may be selected from the nonobese. We mostly anastomosed the LIMAs to the mid segment of the LADs. But sometimes this position may not be visualized so a long LIMA is mostly a must in these operations especially in the learning curve if a distal anastomosis is necessary. Pericardial opening in the initial cases was from where the LAD was seen through the pericardium. But in the later cases the pericardiotomy was done 2-3 cm medial to the phrenic nerve. This made the LAD better visualized when the pericardial traction sutures were held.

When selecting patients for MIDCAB we especially took care that the LADs had a significantly wide diameter. In some of the initial cases patients with small calibration LADs were also included. But after having some difficulties during anastomosis, we did not include those with small LADs. We also selected patients with LADs turning the apex of the heart, which may sometimes be necessary to find the LADs easily because the heart cannot be grossly examined through a small opening.

We did not reoperate on any of the patients for bleeding. Bleeding and blood requirements were lower than those reported [12]. The only patient with prolonged thoracostomy was a patient with severe pleural adhesion. Probably lung injury had occurred during LIMA harvest, and air leakage made the hospitalization longer.

Some authors have reported MIDCAB surgery with epidural anesthesia with no impact on the degree of patient satisfaction. They noted significantly shorter ICU and hospital stay periods, which may result in more efficient use of hospital resources [13].

The MIDCAB operation is more costly than bare metal stenting and more challenging than conventional CABG [14]. On the other hand, a high reintervention rate during the first 6 months equals the initial cost savings of the procedure [15]. For countries like ours which import the stents and which have cheap human resources, MIDCAB may even have equal or lower costs than the stenting procedures.

The MIDCAB can be a useful part of hybrid procedures in patients with multi-vessel disease where a major coronary surgery procedure would not be well tolerated. Complete revascularization can be achieved by a hybrid approach with accompanying percutaneous coronary intervention $[16,17]$. After rescue primary $\mathrm{PCl}$ of the culprit lesion of the circumflex or right coronary artery other than the LAD, patient preference and patients with a high risk for sternotomy-associated problems such as mediastinitis or with reduced life expectancy are some indications for hybrid procedures [16]. On the other hand, deaths have been reported after the surgery owing to unexpected stent thrombosis probably due to surgical manipulation and hemodynamic changes during MIDCAB in addition to inappropriate anticoagulant therapy [18]. As surgeons we think that hybrid procedures in multivessel disease are a compromise between a surgeon and a cardiologist. Actually the point is that the hybrid procedure is the victory of the cardiologist where he is again in the game. Therefore we insist that the above mentioned limited indications should be preserved for hybrid procedures however patients should be imposed on for conventional coronary bypass surgery in the case of preference. To succeed, cooperation of interventional cardiologists, surgeons and anesthesiologists is a must for every miniinvasive hybrid approach [19].

There are three different minimally invasive surgical techniques for left anterior descending (LAD) coronary artery bypass grafting (CABG): port-access surgery (PA-CABG), minimally invasive direct $C A B G$ (MIDCAB) and off-pump totally endoscopic CABG (TECAB). The TECAB is associated with a higher rate 
of early bypass failure and reintervention. No difference between MIDCAB and PA-CABG groups was found. The MIDCAB is concluded to be still the most reliable surgical technique for isolated $L A D$ grafting and the least cost effective [20].

Regarding mortality, most of the clinical trials have demonstrated comparable mortality rates to conventional procedures but lower morbidity and clinical costs in favor of the minimally invasive approach [2]. The MIDCAB in our experience is a very good option for single vessel LAD disease. The study is limited by its retrospective cross-sectional design with a very limited study population. But for surgeons with very limited experience, this study will give some clues to beginners for the MIDCAB procedure.

\section{References}

1. Kolessov VI. Mammary artery-coronary artery anastomosis as method of treatment for angina pectoris. J Thorac Cardiovasc Sug 1967; 54: 535-44.

2. Calafiore AM, Giammarco GD, Teodori G, et al. Left anterior descending coronary artery grafting via left anterior small thoracotomy without cardiopulmonary bypass. Ann Thorac Surg 1996; 61: 1658-65.

3. Zimarino M, Gallina S, Di Fulvio M, et al. Intraoperative ischemia and long-term events after minimally invasive coronary surgery. Ann Thorac Surg 2004; 78: 135-41.

4. Jaffery Z, Kowalski M, Weaver WD, Khanal S. A meta-analysis of randomized control trials comparing minimally invasive direct coronary artery bypass grafting versus percutaneous coronary intervention for stenosis of the proximal left anterior descending artery. Eur J Cardiothorac Surg 2007; 31: 691-7.

5. Fraund S, Herrmann G, Witzke A, et al. Midterm follow-up after minimally invasive direct coronary artery bypass grafting versus percutaneous coronary intervention techniques. Ann Thorac Surg 2005; 79: 1225-31.

6. Cisowski M, Morawski W, Drzewiecki J, et al. Integrated minimally invasive direct coronary artery bypass grafting and angioplasty for coronary artery revascularization. Eur I Cardiothorac Surg 2002; 22: 261-5.

7. Thiele H, Neumann-Schniedewind P, Jacobs S, et al. Randomized comparison of minimally invasive direct coronary artery bypass surgery versus sirolimus-eluting stenting in isolated proximal left anterior descending coronary artery stenosis. J Am Coll Cardiol 2009; 53: 2324-31.

8. Kettering K. Minimally invasive direct coronary artery bypass grafting: a meta analysis. J Cardiovasc Surg 2008; 49: 793-800.

9. Kofidis T, Emmert MY, Paeschke HG, et al. Long-term follow-up after minimal invasive direct coronary artery bypass grafting procedure: a multi-factorial retrospective analysis at 1000 patient-years. Interact Cardiovasc Thorac Surg 2009; 9: 990-4.

10. Holzhey DM, Jacobs S, Mochalski M, et al. Seven-year follow-up after minimally invasive direct coronary artery bypass: experience with more than 1300 patients. Ann Thorac Surg 2007; 83: 108-14.
11. Lewandowski M, Modrzejewski A, Gorący J, Kornacewicz-Jach Z. Myocardial ischaemia due to stenosis of the subclavian artery. Videosurgery Miniinv 2011; 6: 115-20.

12. Arom KV, Emery RW, Flavin TF, Petersen RJ. Cost-effectiveness of minimally invasive coronary artery bypass surgery. Ann Thorac Surg 1999; 68: 1562-6.

13. Kurtoğlu M, Ate S, Bakkaloğlu B, et al. Epidural anesthesia versus general anesthesia in patients undergoing minimally invasive direct coronary artery bypass surgery. Anadolu Kardiyol Derg 2009; 9: 54-8.

14. Magovern JA, Benckart DH, Landreneau RJ, et al. Morbidity, cost, and six-month outcome of minimally invasive direct coronary artery bypass grafting. Ann Thorac Surg 1998; 66: 1224-9.

15. Thiele H, Oettel S, Jacobs S, et al. Comparison of bare-metal stenting with minimally invasive bypass surgery for stenosis of the left anterior descending coronary artery a 5 -year followup. Circulation 2005; 112: 3445-50.

16. Holzhey DM, Jacobs S, Mochalski M, et al. Minimally invasive hybrid coronary artery revascularization. Ann Thorac Surg 2008; 86: $1856-60$

17. Wittwer T, Cremer J, Boonstra P, et al. Myocardial hybrid revascularization with minimally invasive direct coronary artery bypass grafting combined with coronary angioplasty: preliminary results of a multicenter study. Heart 2000; 83: 58-63.

18. Shim JH, Jo WM, Chung WJ, Chung JH. Unexpected stent thrombus after minimally invasive direct coronary artery bypass in hybrid re-vascularisation. Eur J Cardiothorac Surg 2009; 36: 419-21.

19. Haponiuk I, Chojnicki M, Jaworski R, et al. Miniinvasive hybrid closure of multiple muscular ventricular septal defects in a premature infant with novel use of Amplatzer Duct Occluder II - a case report. Videosurgery Miniinv 2011; 6: 33-6.

20. Jegaden $O$, Wautot F, Sassard T, et al. Is there an optimal minimally invasive technique for left anterior descending coronary artery bypass? J Cardiothorac Surg 2011; 25: 37.

Received: 30.04.2012, revised: 9.07.2012, accepted: 20.07.2012. 